\title{
¿Puede el desarrollo ser sostenible, integral y coherente?
}

\author{
Arturo Hernández-Huerta, Octavio Pérez-Maqueo, \\ Miguel Equihua Zamora
}

\begin{abstract}
Resumen: En el Congreso internacional RISC 2017 se reflexionó sobre la posibilidad de lograr un "desarrollo sostenible, integral y coherente". En este artículo nos referimos principalmente al panel de expertos mexicanos que compartieron sus experiencias con nosotros sobre asuntos como el impacto de la agenda internacional sobre la local, la relevancia de la participación de los actores locales y la ocurrencia de incoherencias a lo largo del proceso de diseño y aplicación de las políticas para el desarrollo. Además, se expusieron otras experiencias sobre estos asuntos, que han sido recogidas en este número especial. La conclusión general es que se estima que no sólo es posible articular un desarrollo sostenible, integral y coherente, sino que están emergiendo enfoques y herramientas que favorecen propiciarlo a través de la gestión basada en evidencia y el aprovechamiento del creciente "big data ambiental” que ya está existe.
\end{abstract}

Palabras clave: agenda ambiental, bienestar, bienestar social, big data, capital natural, CPD, i-Gamma, integridad ecosistémica, transversalidad.

En el marco del Programa de Innovación para Integralidad en la Gestión Ambiental del Desarrollo Apoyada en Datos Masivos y Aprendizaje Automatizado (i-Gamma) y el Congreso Internacional del Consorcio para la Investigación Comparada sobre Integración Regional y Cohesión Social (RISC) 2017 realizado en la ciudad de Aguascalientes, Ags., México, se abordó el desafío de conceptualizar un desarrollo sostenible, integral y coherente. Este evento contó además con el respaldo de la red temática CONACyT sobre Socioecosistemas y Sustentabilidad (Socioecos ${ }^{1}$ ). El planteamiento fue una invitación a reflexionar sobre conceptos convergentes, pero también dispares que han venido surgiendo en torno a las opciones para cristalizar un desarrollo en el que los ámbitos económico, cultural y ambiental sean reconocidos y articulados como determinantes de las con- 
diciones de vida de las personas (Fukuda-Parr, 2003; Holden, Linnerud, \& Banister, 2017; Lessmann \& Rauschmayer, 2013). Actualmente existe en el contexto internacional un importante impulso a la concepción de un desarrollo que incluya metas propicias para construir un clima social de mayor seguridad y confianza mutua, que se propone surja de consolidar un orden político democrático con participación ciudadana más eficaz en la gestión y la toma de decisiones públicas. En esta misma concepción interesa incorporar mecanismos que propicien la difusión del bienestar a todos los segmentos de la población, al mismo tiempo que se valora la protección y mejora del hábitat natural para los que lo disfrutan hoy y para quienes lo harán en el futuro (Béjar, 2007).

En la figura 1 esquematizamos tres grandes elementos que subyacen a las diversas posturas en torno al desarrollo. $\mathrm{Al}$ articular lo aprendido comprendemos que el vínculo entre la estructura económica y el crecimiento,

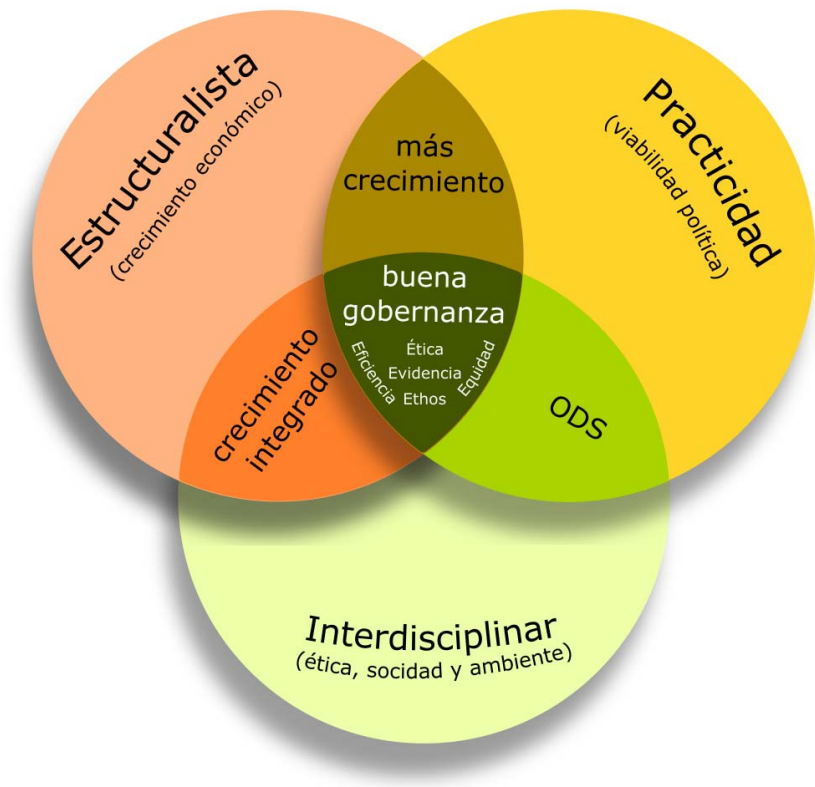

Figura 1. - Las propuestas de desarrollo articulan distintos elementos de acuerdo con las perspectivas conceptuales que se enfaticen. En la figura proponemos tres elementos que percibimos configuran las distintas variantes que actualmente se debaten. Estimamos que la visión del programa "Integralidad Gamma" se encuentra próxima a la noción de un "buena gobernanza" que distinguimos de la simple búsqueda de crecimiento económico (Daly \& Farley, 2011; Mccall \& Dunn, 2012) y estimamos que los elementos de uso del "big data ambiental" que impulsamos contribuye a consolidarlo conceptualmente y a hacerlo operativo. ODS hace referencia a los objetivos de desarrollo sostenible acordados en la ONU. Fuente: Preparación propia. 
la importancia de transitar hacia una visión interdisciplinar del desarrollo y la necesidad de hacerlo posible, son elementos necesarios para la formulación de un "buena gobernanza" que, por tanto, proponemos debe ser: integral, sostenible y coherente. En el contexto internacional las nociones de "transversalidad" o "main streaming" de la biodiversidad y de la dimensión ambiental que ya se impulsan, abonan en la dirección de la integralidad hacia la sostenibilidad. Adicionalmente, en i-Gamma proponemos que la política pública debe operar a partir de evidencia, del acceso a datos y de las capacidades de procesarlos. Por otro lado, apreciamos que el marco normativo y el diseño institucional, para ser eficiente en la conducción hacia la aspiración de sostenibilidad ambiental y social, debe ser alineado de la mejor manera posible para realmente avanzar en la dirección de desarrollo planteado. Esto exige la aplicación de herramientas para analizar objetivamente la coherencia que existe entre los distintos instrumentos de política para el desarrollo que operan internacionalmente y al interior de los países.

En el contexto mundial, las naciones han venido construyendo marcos institucionales en congruencia con las aspiraciones del desarrollo sustentable. Surgen así instrumentos de política que han avanzado para hacer del derecho a un ambiente sano, que se proclama en la declaración de Estocolmo (Brundtland, 1987), un derecho exigible y del concepto de desarrollo sostenible una categoría operacional. Sin embargo, hasta hoy, en la mayoría de los países del mundo existen brechas que limitan al gobierno y a la sociedad para disponer de medios para traducir ese derecho en acciones concretas. Es así que ha venido aflorando un reconocible consenso en torno a la aspiración a que todos los habitantes del planeta somos beneficiarios de la naturaleza al mismo tiempo que responsables de conservar, en la medida de lo posible, la vitalidad y condición funcional de los ecosistemas. En el caso de México estos principios se expresan entre los derechos fundamentales definidos en la Constitución (artículo $4^{\circ}$, párrafo quinto):

Toda persona tiene derecho a un medio ambiente sano para su desarrollo y bienestar. El Estado garantizará el respeto a este derecho. El daño y deterioro ambiental generará responsabilidad para quien lo provoque en términos de lo dispuesto por la ley.

Muchos países han adoptado variantes similares en congruencia con los acuerdos internacionales. Desde luego, cada país tiene el derecho inalienable para regular, en beneficio social, el aprovechamiento de los elementos naturales de su territorio susceptibles de apropiación. Pero, como mencionamos anteriormente, en las concepciones actuales de desarrollo, tal regulación aspira cada vez más a propiciar una distribución equitativa 
de la riqueza pública, cuidar de su conservación, lograr el desarrollo equilibrado del país y el mejoramiento de las condiciones de vida de la población rural y urbana. Esta determinación nacional se extiende al ámbito local, de modo que, por ejemplo, en el caso de México, las Constituciones particulares de cada una de las entidades federativas inciden en la forma en que se distribuye el ejercicio del poder público local y municipal.

El cuerpo normativo nacional es influido por el derecho internacional a través de los tratados, resoluciones o declaraciones de organismos internacionales que impactan así en el interés de la nación. Cada país asimila de manera particular los compromisos asumidos en el ámbito internacional. Por ejemplo, en México, los tratados internacionales, una vez suscritos por el presidente de la República y aprobados por el Senado, se interpretan como una norma suprema de la Unión Federal. En este sentido, las Constituciones y Leyes Municipales no deben contener disposiciones en contrario a dichos tratados. En tales condiciones los tratados en materia ambiental son potencialmente una fuerza relevante al interior del país que surge de la intención global de dar cauce a las relaciones entre Estados soberanos del planeta para alentarlos a proteger y restaurar la biosfera.

En este contexto, la política ambiental surge de un conjunto de ideas, opiniones, principios, teorías y criterios personales de los distintos estudiosos del medio ambiente, de los actores locales que defienden los valores de su entorno y de las normas jurídicas locales e internacionales en la materia. Sus aportaciones impactan en la configuración de los instrumentos de política y eventualmente en la puesta en práctica de la política ambiental. En línea con estos planteamientos, nos preguntamos con un grupo de especialistas mexicanos en el ámbito del "Congreso RISC":

(1) ¿Cómo emergen y de dónde se inspiran las prioridades en las agendas de política ambiental y como se decide sobre su financiamiento?

(2) ¿Existen mecanismos para asimilar las inquietudes sociales locales que buscan evitar el daño ambiental en su entorno o dominan los intereses de los acuerdos internacionales?

(3) ¿Qué tanto corresponde la intención normativa con la práctica en la instrumentación y monitoreo de la política ambiental?

(4) Si lo importante en el desarrollo es el bienestar de las personas ¿cómo se puede lograr esta intención que hace obvio la armonía necesaria entre ambiente y desarrollo económico?

Por otra parte, el avance tecnológico ha hecho posible la adquisición de datos sobre diversos aspectos del ambiente a gran escala y con gran nivel de detalle desde el espacio ${ }^{2}$ y sobre el terreno (por ejemplo, en México el Inventario Nacional Forestal y de Suelos: INFyS). Hoy es posible tener 
una estimación del número de árboles que existen en el planeta y monitorear como está cambiando la cobertura forestal en él (Crowther et al., 2015; Hansen et al., 2013). En el caso de México, con recursos como estos se ha desarrollado el sistema MAD-MEX (Gebhardt et al., 2014), que permite monitorear el cambio en cobertura de los distintos tipos de ecosistemas y usos del terreno a nivel nacional y con una periodicidad anual. También se han desarrollado modelos que permiten estimar el cambio en la condición funcional de los ecosistemas del país a través de un "índice de integridad ecosistémica (Equihua Zamora et al., 2014; Garcia-Alaniz et al., 2017) es decir su integridad. En este capítulo mostramos que es posible desarrollar instrumentos operativos para cuantificar la "integridad ecológica" y desarrollar normas viables para gestionar la intervención humana en los ecosistemas y favorecer la conducción del desarrollo a lo largo de rutas de sustentabilidad. Para ello se requiere lo siguiente: 1, con resolución de 1 $\mathrm{km}^{2}$. El acceso a este tipo de recursos, una especie de "big data ambiental", ofrecen el potencial de incorporar la dimensión ambiental en el diseño y evaluación de la política pública con un enfoque basado en evidencia. Las virtudes y dificultades de estas capacidades analíticas apenas están empezando a ser valoradas. Actualmente existe una gran brecha entre estas capacidades científico-tecnológicas y su comprensión y uso en la gestión de la política pública.

A partir de la conversación con los expertos mexicanos que participaron en el evento en torno a las preguntas que enunciamos anteriormente, emergieron tres asuntos con bastante claridad. El primero fue la discusión en torno a cómo se configura la agenda ambiental, el segundo como se canaliza la participación social y su impacto en la definición de la agenda nacional y el tercero la apreciación de que los instrumentos de desarrollo vigentes no son eficientes para efectivamente propiciar un desarrollo ambientalmente sustentable por presentar incoherencias a múltiples niveles. A continuación, hacemos un recuento de la discusión en torno a estos tres temas, pero además estas inquietudes se aprecian en las contribuciones contenidas en este número especial.

\section{Definición de la agenda ambiental}

En México las prioridades en las agendas de política ambiental se establecen cada seis años, con la elaboración del Plan Nacional de Desarrollo (PND) y los Programas sectoriales, que derivan de éste. En la elaboración del PND sexenal influyen las prioridades identificadas por el equipo de transición, los acuerdos políticos de las campañas electorales y los acuerdos internacionales. En sí, no se percibe que exista una reflexión clara sobre 
la definición nacional de las prioridades ambientales desde una visión a largo plazo. El gobierno en turno es quien define el grado de importancia para cada una de las temáticas identificadas, que emergen en su mayoría de la agenda internacional. Quizás por lo mismo se mantienen prácticamente inalterables durante toda la gestión del gobierno. En la elaboración de los instrumentos de gestión, como los programas sectoriales, casi no se considera un diálogo intersectorial para tener una política pública integradora. Esta rigidez y falta de transversalidad acaba marginalizando la temática ambiental.

Los acuerdos internacionales (por ejemplo, la Convención Marco sobre Cambio Climático - UNFCCC ${ }^{3}$ o la de Convención sobre Biodiversidad - CBD ${ }^{4}$ o la agenda 2030 para el Desarrollo Sostenible - SDG ${ }^{5}$ influyen en la identificación de las prioridades ambientales, pero suele faltar el soporte institucional necesario para cumplir con esos acuerdos. Primero porque las estructuras operativas son demasiado rígidas y no se ajustan a procesos dinámicos, y porque quienes asignan el presupuesto operativo no necesariamente están al tanto de esos acuerdos y de su dinámica. En general, se busca compaginar los temas ambientales de interés, para cumplir con los convenios internacionales y los programas nacionales. $\mathrm{Si}$ bien los convenios internacionales adquieren el nivel de ley y se vuelven vinculantes, esto no necesariamente implica su cumplimiento si se carece de los instrumentos para hacerlo.

Con respecto a los acuerdos internacionales, falta congruencia entre los compromisos que su aceptación implica y la forma como se asimilan en las políticas nacionales. Aun cuando son vinculantes, las acciones para su implementación están sujetas a la interpretación y valoración de las autoridades nacionales. Además, su operación está supeditada a los recursos que decida asignar el país para su puesta en práctica. Con frecuencia se firman los protocolos sin comprometer o asegurar los recursos necesarios para su cumplimiento, pues en realidad se visualizan como una presión externa. De esta manera no se propicia correspondencia entre la intención normativa, su instrumentación y el monitoreo de la política ambiental. Aunque hay diversidad de instituciones y criterios para medir el desempeño de la política, hace falta realizar una evaluación de los impactos de los instrumentos de manera realista. Las visiones prevalecientes en las distintas instancias gubernamentales suelen ser divergentes y dominadas por una visión a corto plazo. La instrumentación suele estar limitada por la práctica de generar una estructura operativa dotada de capacidades insuficientes.

El financiamiento para las prioridades de la agenda de política ambiental se decide cada año. El poder ejecutivo propone las partidas presupuestales, pero el congreso nacional y los congresos estatales determinan la asignación final del presupuesto. Esto origina ciertas incoherencias 
entre prioridades, recursos asignados y metas a alcanzar. Por lo tanto, en la práctica, la visión y la voluntad política del gobierno en turno es fundamental para definir la prioridad de la agenda ambiental y su financiamiento, lo que resulta generalmente en la priorización de asuntos de corto plazo y un muy limitado interés en el largo plazo. En algunos casos los efectos de los desastres naturales han sensibilizado a los gobernantes sobre la importancia de considerar el componente ambiental y de asignarle recursos a la previsión y desarrollo de capacidades para reducir la vulnerabilidad. En otros la academia participa en la planeación de la política ambiental y existen ejemplos de su intervención en algunos estados del país. En resumen, los instrumentos internacionales han inducido un cambio de política ambiental; sin embargo, la implementación es frecuentemente superficial e ineficiente como resultado de incoherencias de varios tipos entre los instrumentos de política que finalmente intervienen en su adopción plena. Otros ejemplos de esta situación se analizan en algunas de las contribuciones incluidas en este número especial, particularmente la de Vázquez y Rivera ("Oportunidades del Acuerdo de Cooperación Ambiental de América del Norte en la era post-TLCAN"). Desde otra perspectiva, la contribución de Zenck, Urrutia y Ríos de este número especial analiza estos aspectos en el caso de "El desarrollo sostenible como política pública: Caso Gobierno Autónomo Descentralizado de Quito".

\section{Participación social}

En México existen mecanismos institucionales y marcos jurídicos para asimilar las inquietudes sociales locales sobre el tema ambiental, pero en general son incipientes. En agosto de 2017 se establecieron lineamientos para impulsar mecanismos de participación ciudadana en las dependencias de la administración pública federal. Todas las secretarías de gobierno cuentan con canales y mecanismos de participación social. Entre todas las dependencias federales, el sector ambiental es el que oferta y cubre el mayor espectro de variantes para la participación, principalmente en los temas de áreas naturales protegidas (93 consejos asesores) y gestión del agua (26 consejos de cuenca). Sin embargo, los especialistas consideran que no son suficientes y que, por ser básicamente de tipo informativo y consultivo, no suelen ser mecanismos proactivos. Esto aunado a que en México el volumen de participación ciudadana es todavía muy bajo, lo cual se atribuye a la falta de confianza hacia las instituciones y al limitado acceso a la información (Serrano Rodríguez, 2015). Por consiguiente, es claro que falta incentivar más procesos participativos para ampliar las capacidades que conduzcan a una mejor identificación de prioridades. En este mismo sen- 
tido aún es limitada la integración con otros sectores, a pesar del reconocimiento de la importancia de transversalizar ("mainstream") la dimensión ambiental. Tal es el caso de brecha entre las agendas del sector agropecuario y el ambiental. Las redes sociales funcionan como espacios informales para diseminar inquietudes sociales sobre medio ambiente. En tanto que el gobierno federal cuenta con la herramienta digital "participa ${ }^{6 "}$, para canalizar la participación ciudadana, pero está enfocado principalmente a dar información y realizar consultas y encuestas. La atención a los aspectos socio participativos es un asunto urgente ya que los intereses sociales divergentes favorecen la conflictividad, por lo que es necesario crear procesos de diálogo para evitar la polarización e integrar elementos de conservación y uso sustentable en los sectores productivos.

Las inquietudes sociales también se asimilan y gestionan a través de los partidos políticos, consejos consultivos, consejos técnicos y comisiones. Formalmente se plasman en documentos diagnósticos, como es el caso de los 16 estudios de estado y las 10 estrategias estatales para la conservación y uso sustentable de la biodiversidad que se han venido desarrollando en México. Aunque hay varios espacios de participación ciudadana institucionalizada, su operación no necesariamente tiene el respaldo de un marco legal. En consecuencia, carecen de presupuesto y son de corto aliento. En el caso de México, la falta de seguimiento de las propuestas ciudadanas genera la sensación de que existe poco aprecio o reconocimiento a las aportaciones de los actores sociales que se suman a los programas promovidos en la agenda gubernamental, lo que genera incomodidad en la sociedad al sentir que no es tomada en cuenta. Al respecto, la contribución de Larsson incluida en este número especial muestra cómo es que las relaciones sociales contribuyen a la coexistencia de distintas visiones respecto de la coherencia de las políticas públicas para el desarrollo entre sociedad y estado.

Los especialistas coinciden en que no hay todavía instancias regionales o estatales adecuadas como para liderar sistemáticamente la gestión de un desarrollo integral e intervenir en instancias de planeación para la aplicación de los recursos de fomento o que decidan sobre las prioridades en la agenda. En la práctica y en ausencia de instancias de planeación que normen la aplicación de los recursos, se generan ocasionalmente duplicidades y hasta situaciones conflictivas. Bajo estas condiciones es frecuente que salgan ganando los grupos de presión política. No se han podido crear hasta ahora, espacios sociales que influyan en la adecuada aplicación de los recursos destinados a propiciar un desarrollo sustentable, integral y coherente. En términos generales se puede decir que no hay en México, todavía, una apropiación social de la agenda ambiental y de sostenibilidad suficientemente pujante como para generar contrapesos adecuados a la 
acción gubernamental ni para impulsar con mayor fuerza la exigencia de transversalizar ambiente, equidad y prosperidad económica en las políticas para el desarrollo. El artículo de Ortega et al. que se incluye en este número especial muestra empíricamente como ocurre esto, pues ilustra las variaciones que existen en la implementación de la Agenda 21 en México.

\section{Coherencia en las políticas públicas para el desarrollo}

De acuerdo con la experiencia de los especialistas, es posible en México identificar incoherencias a varios niveles entre leyes federales y entre instrumentos de planeación, así como las que emergen del plan nacional y de las agendas sectoriales. También es posible reconocerlas entre las acciones programadas y las correspondientes reglas de operación para su aplicación. Como consecuencia, en opinión de los expertos, los temas de la agenda ambiental se atienden de manera desarticulada y se propicia el clientelismo, de acuerdo con la disponibilidad de recursos que otorgan las dependencias. Para remediar esto se necesita mejorar la coordinación, especialmente para optimizar el uso de los presupuestos. Otra consecuencia de la incompleta institucionalización de la agenda ambiental es que no suele haber seguimiento de las acciones realizadas. A pesar de que, para ser congruentes con la normatividad deben existir las capacidades para operar, faltan estructuras eficaces para cumplir con la normatividad, instrumentar las políticas, evaluar su impacto y dar seguimiento a su desempeño. Los expertos señalan que las instituciones disponen de estructura para generar estadísticas de desempeño administrativo, pero no son claros los mecanismos de aseguramiento de la calidad, lo que hace difícil valorar qué tanto esos datos reflejan la realidad. En el proceso de desarrollo de países con economías emergentes, la diversificación de las cadenas productivas podría ser una gran oportunidad para incluir criterios de sustentabilidad, pero estas cadenas no suelen estar bien mapeadas en términos de sus implicaciones ambientales. En general, estiman los especialistas, no se ha propiciado la adopción social de los valores de la sostenibilidad, en la práctica el enfoque dominante es el reactivo con pocos o nulos esfuerzos para recuperar, en todo caso, las lecciones aprendidas.

\section{Aprendizajes}

A pesar de los casi 30 años de debate sobre sustentabilidad y los resultados de la evaluación del milenio (Millenium Ecosystem Assessment, 2005), el alcance de la proposición de que el desarrollo depende del capital natural 
no es tan obvia para los tomadores de decisión ni para el público general. No obstante, la evidencia científica en favor de este planteamiento va en aumento. Los análisis realizados por el IPCC ${ }^{7}$ en el marco del fenómeno de cambio climático son un ejemplo de ello, y lo mismo va ocurriendo con la CBD. Un ejemplo específico lo produjeron Costanza et al. (2008) quienes comprobaron el papel que juega el capital natural (ocurrencia de humedales conservados), en reducir los costos económicos (valor de los daños producidos), que se originaron en la costa Este de los Estados Unidos. El uso de la enorme masa de información que se ha venido acumulando debería ser propicia para ofrecer evidencias más concluyentes al respecto e incluso incorporarla en la práctica cotidiana de toma de decisiones. Por lo tanto, es urgente fomentar su uso en forma más extensiva e involucrar la interpretación de diversos actores. Las instancias gubernamentales necesitan generar mejores y más confiables indicadores de progreso y más precisas definiciones de éxito, así como considerar las expectativas de los diferentes actores para desarrollar una cultura de diseño y conducción de la política pública apoyada en evidencia.

En respuesta a la pregunta que motivó la reflexión sobre la posibilidad de que el desarrollo pueda ser sostenible, integral y coherente el grupo de especialistas mexicanos invitados al Congreso RISC y el público participante debatió apasionadamente la idea, en este debate quedó claro que el desarrollo actual es en gran parte coherente con un desarrollo que no es sustentable ni integral. Pudimos apreciar que hay varios puntos que deben ser atendidos si queremos transitar por la ruta del desarrollo sostenible, integral y coherente. También se hizo evidente que la empresa llevar a cabo estas tareas será complicada pero también se estimó que es posible y que bien vale la pena intentarlo. Para hacerlo tendremos que lograr construir una visión compartida en donde se reconozca simplemente que el bienestar de la sociedad depende en gran medida del capital natural. También será necesario reconocer que el modelo basado principalmente en maximizar el crecimiento económico ha propiciado graves problemas para la convivencia social e incluso que está poniendo en entredicho la sobrevivencia misma del género humano en el planeta. En este sentido, el desafío es encontrar formas eficientes de aprovechar nuestro capital natural de manera más equitativa y sustentable.

Los avances tecnológicos y científicos logrados, la enorme capacidad actual de adquirir datos sobre el estado de los ecosistemas y su evolución ofrecen esperanza por su enorme potencial. Gobernantes y ciudadanos debemos redoblar esfuerzos para incorporar estas capacidades en la convicción de diseñar la política pública para el desarrollo y el cuerpo institucional que la conduce de manera coherente e integral. Como ejemplo de esto, en este número especial Benítez, Alvarado-Castillo, Palestina, Cortés, 
Williams, y Acosta nos ofrecen un análisis sobre los beneficios proporcionados por los cinturones verdes y los desafíos para lograr acuerdos entre ciudadanos y autoridades que deben atenderse para preservar la vegetación remanente en la Ciudad de Xalapa y con ello promover el bienestar de sus habitantes. Desde otra vertiente y dentro del foro de liderazgo en este número especial, Arriaga-Jiménez, Pérez-Díaz, y Pillitteri nos comparten un aprendizaje obtenido desde una aproximación etnográfica, sobre las consecuencias negativas de la pérdida de biodiversidad sobre el conocimiento ecológico tradicional y el lenguaje en la Sierra Mixe del Estado de Oaxaca. México.

\section{AGRADECIMIENTOS}

Agradecemos a Georgina Alcántara López, Hesiquio Benítez Díaz, Héctor Hernández Andrade y Miguel Ángel Velázquez su generosa disposición a compartir con nosotros su experiencia de primera mano en la ejecución de la política pública en México, así como su amplio conocimiento en la materia. Agradecemos a la red SocioEcos su respaldo académico y, junto con CONACyT, el apoyo económico que sumado a la aportación de RISC hicieron posible la realización del evento. Un agradecimiento especial al Centro de Investigación y Docencia Económica (CIDE) que nos recibió en su unidad de Aguascalientes durante la realización del Congreso RISC.

ARTURO HERNÁNDEZ-HUERTA trabaja en la red de ambiente y sustentabilidad del Instituto de Ecología, AC (Inecol). Se interesa en la educación ambiental y enseñanza de las ciencias, entre cuyas colaboraciones destaca el Programa de Aplicación de los Sistemas de Enseñanza Vivencial e Indagatoria de las Ciencias del Estado de Veracruz (PASEVIC), y la elaboración del plan de estudios para: “Educación ambiental para la sustentabilidad en Veracruz", en las escuelas de nivel secundaria de Veracruz. Promueve la ciencia ciudadana, especialmente con la plataforma iNaturalist. Ha intervenido en más de sesenta estudios medioambientales tanto en el sector privado como en el público. Email: arturo.hernández@inecol.mx

OCTAVIO PÉREZ-MAQUEO trabaja en la red de ambiente y sustentabilidad del Instituto de Ecología A.C. Está interesado en evaluar la integridad de los ecosistemas y comprender su relación con el bienestar humano. Ha trabajado en la evaluación de los servicios de los ecosistemas y en la valoración de humedales costeros para la protección de huracanes. Ha coordinado o participado en más de treinta estudios medioambientales tanto en el sector privado como en el público. Email: ocyavio.maqueo@inecol.mx

MIGUEL EQUIHUA ZAMORA trabaja en la red de ambiente y sustentabilidad del Instituto de Ecología, AC (Inecol). Su investigación gira en torno a la compren- 
sión del cambio ambiental inducido por las actividades humanas, sus factores detonantes, magnitud de la transformación y proyección de las tendencias de los cambios. Como parte de estas inquietudes se interesa en el desarrollo de modelos que den cuenta del estado funcional de los ecosistemas que puede caracterizarse como integridad ecosistémica. Con base en esta concepción también se interesa en el análisis del vínculo entre la condición de los ecosistemas y las aspiraciones de desarrollo. Email: miguel.equihua@inecol.mx

\section{NOTES}

1. http://www.redsocioecos.org/

2. http://climate.nasa.gov/nasa_science/missions

3. http://unfccc.int/

4. http://www.cbd.int/

5. http://sustainabledevelopment.un.org/

6. https://www.gob.mx/participa

7. http://ipcc.ch/

\section{REFERENCIAS}

Béjar, R. C. (2007). El nuevo enfoque integrador del desarrollo económico latinoamericano. Boletín Económico de ICE, 2904, 23-44.

Brundtland, G. (1987). Our common future. Report of the World Commission on Sustainable Development. UN, Geneva (Vol. 208). doi: 10.1080/07488008808408783

Costanza, R., Pérez-Maqueo, O., Martínez, M. L., Sutton, P., Anderson, S. J., Mulder, K. (2008). The value of coastal wetlands for hurricane protection. AMBIO: A Journal of the Human Environment, 37(4), 241-248. doi: 10.1579/ 0044-7447(2008)37[241:TVOCWF]2.0.CO;2

Crowther, T. W., Glick, H. B., Covey, K. R., Bettigole, C., Maynard, D. S., Thomas, S. M., Bradford, M. A. (2015). Mapping tree density at a global scale. Nature, 525(7568), 201-205. doi: 10.1038/nature14967

Daly, H. E., \& Farley, J. (2011). Ecological economics: Principles and applications (2nd ed.). Washington, DC: Island Press.

Equihua-Zamora, M., García-Alaniz, N., Pérez-Maqueo, O., Benítez-Badillo, G., Kolb, M., Schmidt, M., Equihua-Benítez, J., Maeda, P. \& Álvarez-Palacios, J.L. (2014). Integridad ecológica como indicador de la calidad ambiental. In C. González-Zuarth, A. Vallarino, A. Low-Pfeng, \& J. C. Pérez (Eds.). Bioindicadores: guardianes de nuestro futuro ambiental. México, DF: Ecosur, INECC. Retrieved from ECOSUR, INECC

Fukuda-Parr, S. (2003). The human development paradigm: Operationalizing Sen's ideas on capabilities. Feminist Economics, 9(2-3), 301-317. doi: 10.1080/ 1354570022000077980

Garcia-Alaniz, N., Equihua, M., Pérez-Maqueo, O., Equihua Benítez, J., Maeda, P., Pardo Urrutia, F., Flores Martínez, J., Villela Gaytán, S., \& Schmidt, M. (2017). 
The Mexican national biodiversity and ecosystem degradation monitoring system. Current Opinion in Environmental Sustainability, 26-27 (Open issue, part II), 62-68. doi: 10.1016/j.cosust.2017.01.001

Gebhardt, S., Wehrmann, T., Ruiz, M. A. M., Maeda, P., Bishop, J., Schramm, M., Schmidt, M. (2014). MAD-MEX: Automatic wall-to-wall land cover monitoring for the Mexican REDD-MRV program using all Landsat data. Remote Sensing, 6(5), 3923-3943. doi: 10.3390/rs6053923

Hansen, M. C. C., Potapov, P. V., Moore, R., Hancher, M., Turubanova, S. A. A., Tyukavina, A., Townshend, J. R. G. R. G. (2013). High-resolution global maps of 21st-century forest cover change. Science, 342(November), 850-854. doi:10.1126/science.1244693

Holden, E., Linnerud, K., \& Banister, D. (2017). The imperatives of sustainable development. Sustainable Development, 25(3), 213-226. doi: 10.1002/sd.1647

Lessmann, O., \& Rauschmayer, F. (2013). Re-conceptualizing sustainable development on the basis of the capability approach: A model and its difficulties. Journal of Human Development and Capabilities, 14(1), 95-114. doi: 10.1080/19452829.2012.747487

Mccall, M. K., \& Dunn, C. E. (2012). Geo-information tools for participatory spatial planning: Fulfilling the criteria for "good" governance? Geoforum, 43(1), 81-94. doi: 10.1016/j.geoforum.2011.07.007

Millenium Ecosystem Assessment. (2005). Ecosystems and human well-being: Synthesis. Washington, DC: Island Press.

Serrano Rodríguez, A. (2015). La participación ciudadana en México. Estudios Políticos, 34(enero-abril), 93-116. doi: 10.1016/j.espol.2015.05.001

\title{
Can development be sustainable, comprehensive and coherent?
}

\section{Arturo Hernández-Huerta, Octavio Pérez-Maqueo, Miguel Equihua Zamora}

\begin{abstract}
At the RISC 2017 International Congress, we reflected on the possibility of achieving a "sustainable, integral and coherent development." We primarily report here on the panel of Mexican experts who shared their experiences on issues such as the impact of the international agenda on the local policy priorities, the relevance of the participation of local stakeholders and the occurrence of inconsistencies throughout the process of design and implementation of development policies. In addition, other experiences were presented on these issues, some of which are included in this special issue. The general conclusion was that not only is it possible to articulate a sustainable, integral and coherent development but also that approaches and tools are already emerging that favor it through an evidence-based policy management and the use of the growing "environmental big data" that already exists.
\end{abstract}

Keywords: big data, ecosystem integrity, environmental agenda, natural capital, PCD, wellbeing 


\section{Le développement peut-il être durable, intégral et cohérent?}

\section{Arturo Hernández-Huerta, Octavio Pérez-Maqueo, Miguel Equihua Zamora}

Résumé : Lors du congrès international Consortium pour la Recherche comparative sur l'intégration régionale et la cohésion sociale (RISC) 2017, organisé en coopération avec le programme d'innovation pour l'intégrité dans la gestion de l'environnement pour le développement et soutenu par des données massives (big data) et un apprentissage automatisé (i-Gamma), nous avons réfléchi à la possibilité de parvenir à un "développement durable, intégral et cohérent". L'événement a ouvert de multiples opportunités de discussions sur le sujet, mais cette introduction est basée sur le panel d'experts mexicains qui ont partagé leurs expériences avec nous sur des questions telles que l'impact de l'agenda international à l'échelle locale, la pertinence de la participation des acteurs locaux et le surgissement d'incohérences tout au long du processus de conception et de mise en œuvre des politiques de développement. Nous ferons également référence à d'autres expériences présentées autour de ces questions, en mettant l'accent sur les contributions de ce numéro spécial. En conclusion générale, nous pensons qu'il n'est pas seulement possible d'articuler un développement de manière durable, intégrale et cohérente, mais que des approches et des outils sont déjà en train d'émerger et favorisent une gestion fondée sur des données probantes et l'utilisation des "données environnementales à grande échelle » déjà existantes.

Mots-clés : agenda environnemental, bien-être, bien-être social, Big data, capital naturel, Cohérence des politiques de développement (CPD), intégrité de l'écosystème, transversalité 